\title{
Les Déterminants du Concept de Soi Dans un Contexte Africain : Cas des Élèves de la Région d'Abidjan en Côte d'Ivoire
}

\author{
Aka Flaubert Koukougnon \\ Département des sciences de l'éducation, \\ Ecole Normale Supérieure, Abidjan, Côte d'Ivoire
}

\section{Résumé}

Cette étude vise à montrer l'importance du Statut socioéconomique(SSE) de la famille des élèves et des conditions de fréquentation dans la détermination du concept de soi en milieu Africain. Pour ce faire, elle propose d'analyser les liens de causalité entre les facteurs du milieu socioéducatif des élèves et l'évaluation du concept de soi. Elle part du constat que si les facteurs du milieu ont une influence sur les performances des élèves (Coleman, 1966) et qu'au surplus, les performances influencent la définition du concept de soi (Chanal, 2005) alors les facteurs du milieu devraient influer sur la détermination du concept de soi. Pour vérifier cette hypothèse, une enquête quantitative et qualitative a été menée sur 200 élèves des classes de CE2 et CM1 de 10 écoles de la région d'Abidjan en Côte d'ivoire. Les résultats montrent que les facteurs du milieu socioéducatif agissent indépendamment ou concomitamment ou cumulativement sur la détermination du concept de soi.

Mots-clés : Facteurs du milieu, Fréquentation, déterminant, concept de soi, élèves, abidjan 


\title{
The Determinants of the Concept of Self in an African Context: A Case Study of the Students in the Region of Abidjan in Côte d'Ivoire
}

\author{
Aka Flaubert Koukougnon \\ Département des sciences de l’éducation, \\ Ecole Normale Supérieure, Abidjan, Côte d’Ivoire
}

\begin{abstract}
This paper focuses on showing the importance of the social and economic status of family and the conditions of frequentation in the determination of the concept of self in an African environment. In order to do this, it proposes to analyze the causal links between the factors in the socioeducational environment of students and the evaluation of self-concept. It is based on the observation that environmental factors have an influence on students' performance (Coleman, 1966). Performance influences the definition of self-concept (Chanal, 2005), and environmental factors also influences the determination of the self-concept. To confirm this hypothesis, a quantitative and qualitative survey was conducted on 200 students in CE2 and CM1 from 10 schools in the Abidjan region of Côte d'Ivoire. The results demonstrated that socio-educational factors act independently, concomitantly, or cumulatively in the determination of the self-concept.
\end{abstract}

Keywords: Environmental factors, Frequentation, Determinant, Self-concept, pupils, Abidjan

\section{- Introduction}

Le concept de soi apparait comme un terme générique qui inclut différents aspects de la perception de soi. Il est perçu comme « la totalité des pensées et des sentiments de l'individu ayant comme référence lui-même en tant qu'objet » (Rosenberg, 1965). Il se forme à travers les expériences avec l'environnement (Shavelson, 1976) comme par exemple les comparaisons sociales, les attributions causales et même les effets miroirs. Ainsi, élaboronsnous des conceptions de nous-mêmes dans différents domaines où nous acquérons de l'expérience.

En termes plus larges, on peut affirmer avec Shavelson (1976) que le concept de soi est « une perception de l'individu sur lui-même. Elle est 
présumée influencer les manières avec lesquelles l’individu agit, et ses actions influencent en retour la manière dont l'individu se pensait »

C'est un construit :

- organisé et structuré autour de la famille, des amis, de l’école, du travail... variable selon les individus.

- multidimensionnel qui associe le scolaire, le physique, le relationnel, etc.

- hiérarchisé en soi scolaire et non scolaire.

- stable généralement mais qui devient changeant quand on descend dans les ramifications de l'échelle hiérarchique.

- développemental c’est-à-dire qui croît avec l'âge et au fur et à mesure qu’on progresse dans la scolarité.

- évaluatif et descriptif quand l’individu décrit sa nature et lui attribut un jugement de valeur (je suis littéraire; je suis un très bon littéraire).

- différenciable de la corrélation de la réussite plus au concept de soi spécifique que général.

Pour bien comprendre le concept de soi des élèves et la relation causale qu'il essaie d'établir entre celui-ci et l'environnement, il faut savoir le distinguer de l'estime de soi.

Selon Coopersmith (1967) l'estime de soi est une « évaluation que l'individu fait et qu'il entretient habituellement: elle exprime une attitude d'approbation ou de désapprobation et indique le degré selon lequel il se croit lui-même capable, important, en pleine réussite et digne. Bref l'estime de soi est un jugement personnel de mérite qui s’exprime dans des attitudes que l'individu véhicule vers les autres par la communication verbale et par d'autres comportements expressifs ».

Comme on peut le noter, alors que le concept de soi est un jugement de valeur concernant les capacités et aptitudes que l'individu possède dans différents domaines (je suis un bon élève; j’ai de bonnes notes), l'estime de soi est une évaluation affective de soi-même qui est plus globale et qui renvoie à la manière dont l'individu se sent vis-à-vis de lui-même (je suis heureux; je suis chanceux).

L'estime de soi peut être une évaluation globale du concept de soi (Shavelson, 1976). Elle n’est pas attachée à un domaine particulier et sa valeur peut être négative alors que l'individu à un concept de soi positif dans un ou plusieurs domaines. Dans ce cas l'estime de soi aurait été influencée par les quelques domaines négatifs du concept de soi. C'est pourquoi des chercheurs comme Marsh (1993) pense que l'estime de soi n'est pas un construit utile car trop subjectif.

Par contre, l’efficacité personnelle traduit comme «...les jugements des individus sur les capacités à organiser et à exécuter le déroulement d'une 
action requise pour atteindre les performances désirées » (Bandura, 1986), représente les attentes des individus de ce qu'ils pensent pouvoir réussir dans des situations données. Selon cette approche, les perceptions relatives aux croyances des individus à pouvoir réaliser une tâche particulière serait un déterminant plus important que l'évaluation de leur propre compétence dans la réussite. En d'autres termes, le sentiment d'efficacité personnelle serait plus important que le concept de soi pour l'accomplissement d'une tâche particulière (Bong, 1998). En outre, si le concept de soi s’oriente sur le présent (je suis bon en lecture), l'efficacité personnelle s'oriente vers le futur (j'ai des chances de réussir en maths). Ainsi, alors que le concept de soi inclut de manière générale les notes, les scores, la motivation et même l'anxiété (Bong, 1998), l'efficacité personnelle s'intéresse aux fixations de buts, à la persévérance, aux efforts et performances spécifiques à une tâche.

Si nous savons maintenant définir le concept de soi, le distinguer de l'estime de soi et de l'efficacité personnelle, la question des déterminants du concept de soi, c'est-à-dire des situations antécédentes, qui influencent l'évaluation du concept de soi, demeure entière. Certaines études montrent l'influence de l'âge et du sexe qui sont des facteurs invariants (Marsh \& Craven, 1997). D’autres études notamment celles de Chanal (2005) évoque 5 situations antécédentes qui agissent comme facteurs déterminants de l'évaluation du concept de soi. Ce sont: les résultats scolaires, l'autosatisfaction, la comparaison des performances, les jugements d'autrui significatif, l'intérêt pour domaine. La fréquence de la réussite, l'apparence, les habiletés, les jugements des pairs, sont les indicateurs les plus couramment cités comme influençant l'évaluation du concept de soi.

Cependant, les variables et indicateurs liés au statut socioéconomique (SSE) de la famille et des conditions de fréquentation n’ont pas été pris en compte. En effet, la relation entre les variables du milieu familial et les performances scolaires est confirmée depuis la célèbre étude Coleman aux USA en 1966. En outre, une étude menée par Dolata (2000) en Afrique, a identifié les variables du milieu familial, susceptibles d'influencer les performances scolaires. Parmi ceux-ci, on note les dimensions culturelle et matérielle de la famille, la qualité de l'habitat et enfin le revenu.

Qu'en est-il alors de la relation entre les SSE de la famille et le concept de soi. Peut-on considérer le SSE de la famille et subséquemment, les conditions de fréquentation comme facteurs déterminants de l'évaluation du concept soi?

Pour répondre à cette question, cette étude se fixe pour objectif d'analyser le lien de causalité entre les facteurs du milieu, les conditions de fréquentation et l'évaluation du concept de soi. En d'autres termes, il s'agira d'amener les sujet-élèves à justifier l'évaluation du concept de soi, de répertorier les avis, les opinions, les explications pour vérifier l'occurrence des 
déterminants identifiés dans les études antérieures et éventuellement, prouver que les facteurs du milieu familial et des conditions de fréquentation sont tout aussi déterminants dans l'évaluation d'un concept de soi en milieu africain en l'occurrence émise à Abidjan en Côte d'Ivoire.

L'hypothèse émise est que si la relation entre les variables du milieu et les performances scolaires est établie (Coleman, 1966) et qu'au surplus, celle entre les performances scolaires et le concept de soi l'est aussi (Chanal, 2005), alors par effet transitif, on peut affirmer que la relation entre les variables du milieu et le concept de soi, est aussi établie. Par conséquent, le SSE de la famille et les conditions de fréquentation doivent apparaitre comme facteur déterminant l'évaluation du concept de soi.

\section{2-Méthodologie}

\section{La population cible}

Pour vérifier notre hypothèse, notre population d'étude est représentée par 200 élèves de CE2 et de CM2, de 10 écoles des communes de Yopougon et Songon en Côte d'Ivoire. Les élèves, dont l'âge oscille entre 8 et 11ans, ont été choisis de façon aléatoire en ayant respecté la parité du genre et des tranches d'âge. Il faut noter que chacun des sujet-élèves a été préalablement soumis au questionnaire de description de soi (QDS) de Marsh (1988) tel que validé par Dierendonck (2008).

\section{Structure de l'échantillon}

Tableau 1. Répartition de l'échantillon en fonction de l'âge et du sexe

\begin{tabular}{|l|l|l|l|l|l|l|l|l|l|l|}
\hline $\begin{array}{c}\text { Age } \\
\text { Sexe }\end{array}$ & \multicolumn{2}{l|}{ 8 } & \multicolumn{2}{l|}{ 9 } & \multicolumn{2}{l|}{10} & \multicolumn{2}{l|}{11} & \multicolumn{2}{l|}{ Total } \\
\hline Filles & 25 & & 25 & & 25 & & 25 & & 100 & $50 \%$ \\
\hline Garçons & 25 & & 25 & & 25 & & 25 & & 100 & $50 \%$ \\
\hline Total & 50 & $25 \%$ & 50 & $25 \%$ & 50 & $25 \%$ & 50 & $25 \%$ & 200 & $100 \%$ \\
\hline
\end{tabular}

La parité du genre dans les tranches d'âge permet d'éviter à priori les effets du nombre et d'équilibrer les échanges et les opinions dont la prédominance aurait pu être marquée par le sceau de la féminité ou de la masculinité. On pourrait citer par exemple, la question des 'travaux domestiques', qui touche plus les filles que les garçons.

\section{Les outils de recherche}

\section{Guide d'entretien}

Pour optimiser les résultats de l'entretien, nous avons constitué 10 focus group de 20 élèves chacun de façon à intensifier les interactions entre enquêté/ enquêteur dans un entretien semi-directif. Chaque focus group comprenant autant de filles que de garçons. 
L'entretien porte sur les 5 variables de Chanal (2005) et les 2 variables de notre hypothèse (6\&7):

- Les résultats scolaires

- L'auto-satisfaction

- La comparaison des performances

- Le jugement d'autrui significatif

- L'intérêt dans le domaine

- Le SSE de la famille

- Les conditions de fréquentation

L'entretien consiste à recueillir les avis, les explications des élèves quant à l'importance de ces variables dans la détermination du concept de soi dans les domaines académique et non académique et d'identifier avec eux, les indicateurs significatifs.

\section{Questionnaire d'enquête}

Un questionnaire est adressé aux élèves pour rechercher l'importance des variables et items de variables dans la détermination d'un concept de soi. Ce questionnaire est ainsi résumé:

1. Les variables ci-dessus répertoriées induisent-elles un sentiment de soi négatif et/ou positif ? justifier votre réponse.

2. Les indicateurs ci-dessus répertoriés induisent-ils un sentiment de soi négatif et/ou positif ? justifier votre réponse.

Tableau 1. Variables explicatives

\begin{tabular}{|l|l|l|l|l|}
\hline Variables explicatives & \multicolumn{3}{|c|}{ Cocher et justifier } \\
\cline { 2 - 5 } & $\begin{array}{c}\text { Concept } \\
\text { de soi } \\
\text { négatif }\end{array}$ & Justifier & $\begin{array}{c}\text { Concept } \\
\text { de soi } \\
\text { positif }\end{array}$ & Justifier \\
\hline Résultats scolaires & & & & \\
\hline Auto-satisfaction & & & & \\
\hline Comparaison des performances & & & & \\
\hline Jugement d'autrui significatif & & & & \\
\hline Intérêt dans le domaine & & & & \\
\hline SSE de la famille & & & & \\
\hline Conditions de fréquentation & & & & \\
\hline
\end{tabular}


Tableau 2. Indicateurs des variables

\begin{tabular}{|l|l|l|l|l|}
\hline \multirow{2}{*}{ Indicateurs explicatives } & $\begin{array}{c}\text { Concept } \\
\text { de soi } \\
\text { négatif }\end{array}$ & Justifier & $\begin{array}{c}\text { Concept } \\
\text { de soi } \\
\text { positif }\end{array}$ & Justifier \\
\hline Echec & & & & \\
\hline Réussite & & & & \\
\hline Habiletés & & & & \\
\hline Difficulté & & & & \\
\hline Apparence & & & & \\
\hline Chance & & & & \\
\hline Comparaison aux pairs & & & & \\
\hline Comparaison à soi même & & & & \\
\hline Jugement des enseignants & & & & \\
\hline Jugement des parents & & & & \\
\hline Jugement des pairs & & & & \\
\hline Etat de mon habitat & & & & \\
\hline Nbre de repas/jour & & & & \\
\hline Présence d'électricité & & & & \\
\hline Suivi à domicile & & & & \\
\hline Travaux domestiques & & & & \\
\hline Présence d'eau potable & & & & \\
\hline Distance école/famille & & & & \\
\hline Cantine scolaire & & & & \\
\hline Pratique pédagogique & & & \\
\hline Épreuves d'évaluation & & & \\
\hline
\end{tabular}

\section{Analyse des données}

L'analyse quantitative a consisté à déterminer la fréquence des variables et indicateurs évoqués comme facteur influençant l'évaluation du sentiment de soi, en fonction de l'âge et du sexe.

L'analyse qualitative a consisté à évaluer la pertinence des justifications et à faire un tri en fonction de leur occurrence. 
- Résultats

31- Déterminants du concept de soi POSITIF

Tableau 3. Fréquence des facteurs explicatifs en fonction de l'âge et du sexe

\begin{tabular}{|c|c|c|c|c|c|c|c|c|c|c|c|}
\hline \multirow{3}{*}{$\begin{array}{l}\text { Variables } \\
\text { Explicative } \\
\mathrm{S}\end{array}$} & \multirow{3}{*}{ Indicateurs } & \multicolumn{10}{|c|}{ Concept de soi POSITIF } \\
\hline & & \multicolumn{5}{|c|}{ Filles (\%) } & \multicolumn{5}{|c|}{ Garçons (\%) } \\
\hline & & 8 & 9 & 10 & 11 & $\mathbf{T}$ & 8 & 9 & 10 & 11 & $\mathbf{T}$ \\
\hline \multirow{2}{*}{$\begin{array}{l}\text { Résultats } \\
\text { scolaires }\end{array}$} & Echec & & & & & & & & & & \\
\hline & Réussite & 100 & 100 & 100 & 100 & 100 & 100 & 100 & 100 & 100 & 100 \\
\hline \multirow{4}{*}{$\begin{array}{l}\text { Auto- } \\
\text { satisfaction }\end{array}$} & Habilités & 100 & 100 & 100 & 100 & 100 & 100 & $\begin{array}{l}1 \\
00\end{array}$ & 100 & 100 & 100 \\
\hline & Difficultés & & & 2 & 5 & 1.8 & & & 5 & 8 & 3.2 \\
\hline & Facilités & 100 & 100 & 100 & 70 & & 100 & $\overline{95}$ & 70 & 60 & \\
\hline & Apparence & 25 & 30 & 25 & 25 & & 15 & 10 & 12 & 8 & \\
\hline \multirow{2}{*}{$\begin{array}{l}\text { Comparais } \\
\text { on des } \\
\text { performan } \\
\text { ces } \\
\end{array}$} & $\begin{array}{l}\text { Plus perfor. que } \\
\text { les pairs }\end{array}$ & 100 & 100 & 100 & 100 & & 100 & 100 & 100 & 100 & \\
\hline & Par rapport à soi & 100 & 100 & 60 & 50 & & 80 & 70 & 60 & 60 & \\
\hline \multirow{3}{*}{$\begin{array}{l}\text { Jugement } \\
\text { d'autrui } \\
\text { significatif }\end{array}$} & $\begin{array}{c}\text { Jugement positif } \\
\text { des pairs }\end{array}$ & 100 & 100 & 100 & 100 & & 100 & 100 & 100 & 100 & \\
\hline & $\begin{array}{l}\text { Jugement positif } \\
\text { des enseignants }\end{array}$ & 100 & 100 & 100 & 100 & & 100 & 100 & 100 & 100 & \\
\hline & $\begin{array}{c}\text { Jugements } \\
\text { positif des } \\
\text { parents } \\
\end{array}$ & 100 & 100 & 100 & 100 & & 100 & 100 & 100 & 100 & \\
\hline \multirow{2}{*}{$\begin{array}{l}\text { Intérêt } \\
\text { dans le } \\
\text { domaine }\end{array}$} & Intérêt & 100 & 100 & 100 & 100 & & 100 & 100 & 100 & 100 & \\
\hline & Désintérêt & - & - & - & & & - & - & - & - & \\
\hline \multirow{6}{*}{$\begin{array}{l}\text { SSE de la } \\
\text { famille }\end{array}$} & Type de l'habitat & $\ldots$ & & 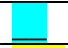 & 5 & &  &  & & 3 & \\
\hline & $\begin{array}{l}\text { Présence } \\
\text { d’électricité }\end{array}$ & 100 & 100 & 100 & 100 & & 100 & 100 & 100 & 100 & \\
\hline & Suivi à domicile & 100 & 100 & 100 & 100 & & 100 & 100 & 100 & 100 & \\
\hline & $\begin{array}{l}\text { Présence d'eau } \\
\text { potable }\end{array}$ & 100 & 100 & 100 & 100 & & 100 & 100 & 100 & 100 & \\
\hline & $\begin{array}{l}\text { Travaux } \\
\text { domestiques }\end{array}$ & -- & -- & 3 & 5 & & - & -- & - & -- & \\
\hline & $\begin{array}{l}\text { Trois repas par } \\
\text { jour }\end{array}$ & 100 & 100 & 100 & 100 & & 100 & 100 & 100 & 100 & \\
\hline \multirow{3}{*}{$\begin{array}{l}\text { Conditions } \\
\text { de } \\
\text { fréquentati } \\
\text { on }\end{array}$} & $\begin{array}{l}\text { Distance école- } \\
\text { domicile }\end{array}$ & $\overline{--}$ &.-- & - & - & & - & $\overline{-1}$ & - & --- & \\
\hline & $\begin{array}{l}\text { Présence de } \\
\text { Cantine scolaire } \\
\end{array}$ & 100 & 100 & 100 & 100 & & 100 & 100 & 100 & 100 & \\
\hline & $\begin{array}{l}\text { Pratique } \\
\text { pédagogique } \\
\text { souple du maître }\end{array}$ & 100 & 100 & 100 & 100 & & 100 & 100 & 100 & 100 & \\
\hline
\end{tabular}


Les résultats montrent qu'il existe quatre groupes d'indicateurs qui interviennent dans la détermination du concept de soi positif, selon leur occurrence:

Groupe 1 (colonnes jaunes): Les indicateurs reconnus par l'ensemble (100\%) des sujets comme facteurs déterminants du concept de soi positif quel que soit l'âge et le sexe, sont : la réussite scolaire, les habiletés, la facilité des épreuves, les jugements positifs d'autrui significatif, l' intérêt dans le domaine, la présence d'électricité à domicile, la présence d'eau potable à domicile, les trois repas journaliers, la présence de cantine à l'école, la pratique pédagogique souple du maître, le suivi pédagogique à domicile du sujet.

Groupe 2 (colonnes vertes): Les indicateurs ayant une influence certaine sur l'évaluation du concept de soi positif mais avec des nuances en fonction du sexe et de l'âge, sont : l'apparence physique (26\% pour les filles et $11 \%$ pour les garçons, en moyenne), la facilité des épreuves (92\% pour les filles et $81 \%$ pour les garçons, en moyenne)

Groupe 3 (colonnes bleues): Les indicateurs ayant très peu d'influence sur la détermination du concept de soi positif, sont : les difficultés dans l'apprentissage (1.8\% des filles et 3.2\% des garçons, en moyenne), le type d'habitat ( $1.2 \%$ et $0.7 \%$ ), les travaux domestiques ( $2 \%$ des filles et $0 \%$ des garçons, en moyenne)

Groupe 4 (colonnes violettes): les indicateurs n'ayant aucune influence sur la détermination du concept de soi positif, sont : l'échec scolaire et la distance Ecole-famille.

Il faut noter que la présence des indicateurs du groupe 1dans l'environnement socioéducatif induit forcément un sentiment de soi positif qu'il soit pris individuellement ou collectivement.

Dans le groupe 2, la fréquence et la répartition des indicateurs montrent que leur prise en compte dépend du sexe et de l'âge des sujets.

En ce qui concerne l'apparence physique, en moyenne 26\% des filles estiment qu'elle influence l'évaluation du concept de soi positif alors que seulement $11 \%$ des garçons lui accordent du crédit.

En ce qui concerne la facilité de l'apprentissage et des épreuves, seuls $92 \%$ des filles et $81 \%$ des garçons pensent qu'elle est génératrice de construits positifs.

Le type d'habitat et les travaux domestiques et les difficultés de l'apprentissage ont une occurrence presqu'inexistante dans la construction d'un concept de soi positif (Groupe 3) alors que la distance école-famille et l'échec scolaire (groupe 4) n'y participent pas du tout. Ces tendances ont été exprimées par des sujets de 10-11ans aussi bien chez les filles que chez les garçons. 


\section{Déterminants du concept de soi NEGATIF}

Tableau 4. Fréquence des facteurs explicatifs en fonction de l'âge et du sexe

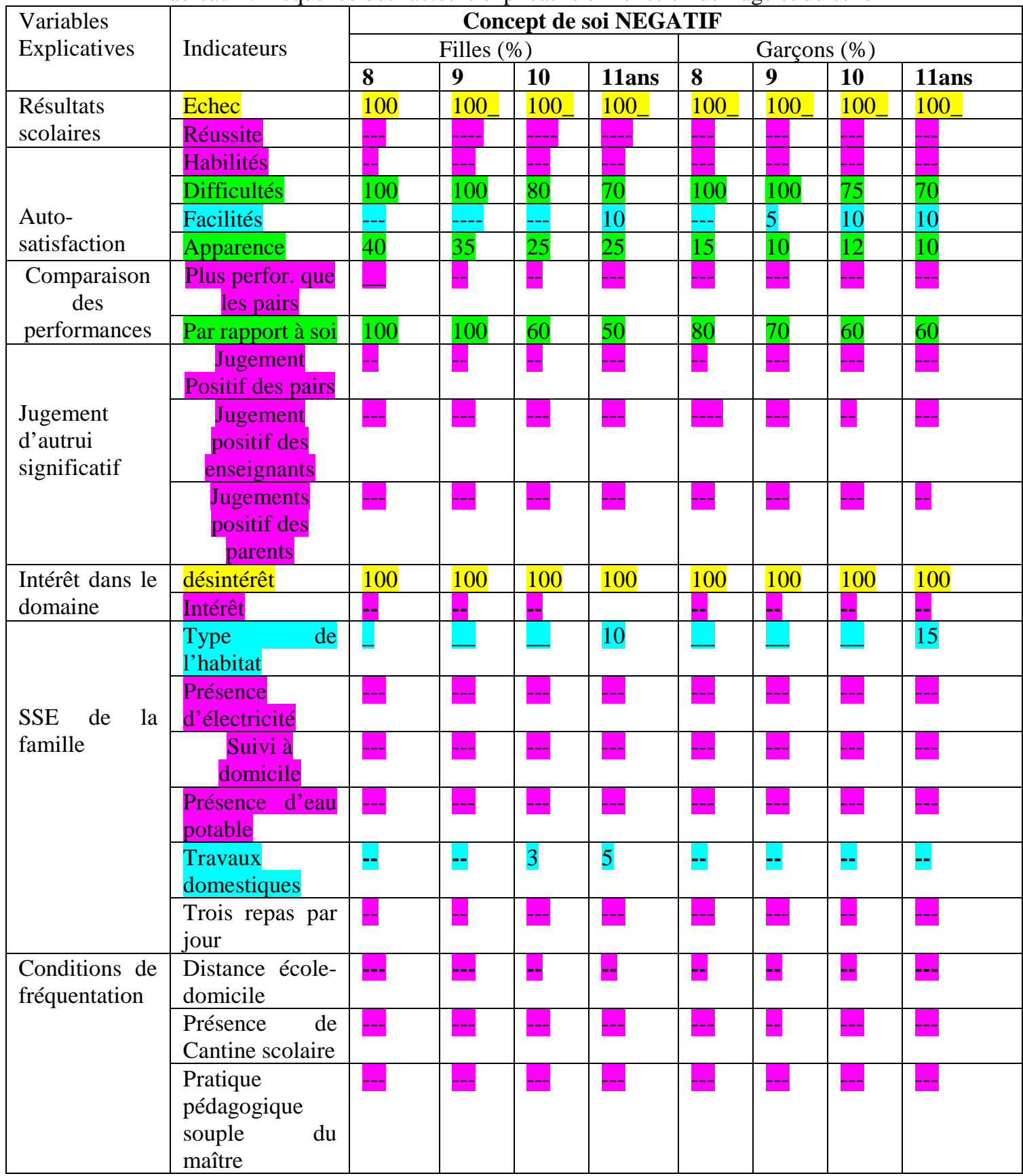

L'analyse des résultats de la détermination du concept de soi négatif montre 4 groupes d'indicateurs: 
Groupe 1 (colonnes jaunes): les indicateurs dont l'influence est confirmée par $100 \%$ des sujets quelque soit l'âge et le sexe, sont : l'échec scolaire et le désintérêt dans le domaine d'étude. Ces indicateurs génèrent forcément un sentiment de soi négatif.

Groupe 2 (colonnes vertes) : les indicateurs très influents mais dont la fréquence varie en fonction de l'âge et du sexe, sont : le jugement par rapport à soi ( $70 \%$ des filles et $70 \%$ des garçons en moyenne), les difficultés des épreuves (87.5\% de filles et, $86.2 \%$ des garçons en moyenne), l'apparence physique (31.2\% des filles et $11.7 \%$ des garçons en moyenne).

Groupe 3 (colonnes bleues) : les indicateurs très peu influents comme le type d'habitat (2.5\% des filles et $6.2 \%$ des garçons) et les travaux domestiques ( $2 \%$ des filles et $0 \%$ des garçons) et la facilité de l'apprentissage ( $2.5 \%$ de filles et $6.2 \%$ des garçons) ont une occurrence qui varie en fonction de l'âge et du sexe.

Groupe 4 (colonnes violettes): les indicateurs qui n'ont pas d'influence sur l'évaluation du concept de soi négatif quelque soi l'âge et le sexe, sont : l'intérêt dans le domaine, la réussite scolaire, les habiletés, les performances comparatives, les jugements positifs d'autrui significatif, la présence d'électricité et d'eau potable, trois repas par jour, etc.,

Il faut d'emblée noter que la récurrence de l'échec et la manque d'intérêt dans un domaine d'étude génèrent forcément un sentiment de soi négatif dans le dit domaine.

En ce qui les jugements par rapport à soi, $70 \%$ de filles et $70 \%$ des garçons ont confirmé leur tendance à générer un sentiment de soi négatif quelque soit l'âge.

En outre, $31 \%$ de filles et $11.7 \%$ des garçons estiment que l'apparence physique induit un sentiment de soi négatif.

Selon $10 \%$ des filles de 11 ans et $13 \%$ des garçons la facilité des épreuves peut être l'origine de sentiment de soi négatif.

Enfin $2.5 \%$ des filles et $6.2 \%$ des garçons estiment le type d'habitat peut induire un sentiment de soi négatif alors seulement $2 \%$ le pensent pour les travaux domestiques.

\section{Déterminants et du concept de soi}

Les résultats montrent que les 7 variables identifiés dans les outils d'enquête influencent effectivement la détermination du concept de soi, qu'il soit négatif ou positif.

Certains indicateurs de variables agissent de façon dichotomique ; ce sont par exemple les indicateurs "échec/réussite" de la variable "'réussite scolaire" ou les indicateurs "' positif/négatif' de la variable '’jugement d'autrui significatif'" ou encore les indicateurs 'intérêt/désintérêt' de la variable "'intérêt dans le domaine d'étude" ou enfin des indicateurs "' 
présence/absence” des variables 'sSE de la famille”' et des '’conditions de fréquentation'”.

Les autres indicateurs dépendent de la perception du sujet en fonction du sexe et de l'âge. C'est par exemple le cas des indicateurs “' facilité/difficulté'’ de l'apprentissage ou de l'apparence physique ou encore de la distance école-famille ou enfin des travaux domestiques.

\section{Justifications de l'évaluation du concept de soi par le sujet}

L'analyse des contenus des réponses justifiant l'influence ou non des indicateurs dans la détermination du concept de soi nous ont permis d’identifier 7 situations antécédentes évoquées par les élèves comme ayant une relation causale avec la valeur de l'évaluation du concept de soi.

\section{- la fréquence de la réussite ou d’échec}

Les sujets ayant un concept de soi positif, quelque soit le domaine, ont répondu avoir l'habitude d’y réussir, sans évoquer une raison particulière. Il en est de même de ceux qui ont un concept de soi négatif. Ils disent avoir l'habitude d’échouer dans ces domaines.

\section{- l'auto- satisfaction}

Les sujets ayant un concept de soi positif l'attribut à leurs efforts et habilités. A contrario, ceux ayant un concept de soi négatif l'attribut à la chance, aux difficultés de la tâche, à divers déficits environnementaux etc., l'auto satisfaction est la variable dont les indicateurs sont les plus nuancés. On constate que les difficultés/facilités de l'apprentissage et/ ou des épreuves ne génèrent pas forcément des construits négatifs/positifs de même que l'apparence physique.

\section{- la comparaison des performances}

Les sujets justifient l'évaluation de leur concept de soi en comparaison de leur performance à celle des pairs ou en comparant leur propre performance dans diverses matières.

\section{- le jugement d'autrui significatif}

Les sujets affirment avoir toujours été considérés comme étant faibles par les enseignants ou par leurs parents ou par les pairs et qu'ils ont fini par s'en tenir à ce jugement.

\section{- l'intérêt pour le domaine}

Les sujets justifient l'évaluation de leur concept de soi par l’intérêt qu’ils portent au domaine d'étude concerné.

\section{- le SSE de la famille}

Les sujets attribuent l'évaluation de leur concept de soi aux conditions de vie en famille notamment à la présence d'un certains nombre d'équipement de commodités comme l'électricité, l'eau potable, le nombre de repas, la présence d'un répétiteur etc., 


\section{- les conditions de fréquentation}

Les sujets attribuent l'évaluation de leur concept de soi à la distance école-famille, à la présence dans l'école de cantine scolaire, au mode de leadership pédagogique du maître, au niveau des évaluations, etc.

\section{Discussion et conclusion}

Il faut d'emblée noter que le statut socioéconomique de la famille, les conditions de fréquentation et les indicateurs qui les caractérisent constituent les principales situations antécédentes qui influencent l'évaluation du concept de soi en milieu africain. Il est vrai que l'étude de Chanal (2005) n'avait pas pris en compte ces deux variables considérant sans doute qu'elles n'étaient pas assez discriminatoires. Si cela peut paraître défendable dans les pays développés, dans les milieux de fortes inégalités notamment en Afrique, les facteurs du milieu et les conditions de fréquentations discriminent les élèves et influencent le sentiment de soi. Ceci est largement prouvé par les résultats de l'étude qui citent les indicateurs de ces variables comme facteurs influençant le concept de soi de l'ensemble des sujets.

Cependant, à l'analyse des situations antécédentes, on note une complexité de sentiments induits par certains indicateurs des variables du SSE de la famille et des conditions de fréquentation notamment le niveau de difficulté/facilité des évaluations, la distance Ecole-famille, le type d'habitat ou les travaux de ménage. Le niveau de difficulté induit pour certains un sentiment de soi positif alors que la facilité induit pour d'autres un sentiment de soi négatif. Cette apparente contradiction est justifiée par l'envie du dépassement de soi dans le premier cas et dans le second, par le refus de la surestimation de soi. Le type d'habitat, quant à lui, n'influence que les sujets en fin de cycle (11ans) parce qu'à cet âge, ils intègrent les valeurs de comparaison alors que les travaux de ménage n'influencent les filles que dans une proportion très réduite (2\%) parce qu'il s'agit d'un vécu quotidien, normalisé comme tel.

En définitive, l'évaluation du concept de soi en milieu scolaire est influencée par 7 déterminants:

1. Les résultats de l'élève lui-même

2. Les résultats des pairs et du groupe-classe

3. Les attentes et la perception de l'enseignant

4. Le SSE de la famille de l'élève

5. Les conditions de fréquentation

6. Les perceptions d'autrui significatif

7. L’intérêt de l'élève pour le domaine.

Ces déterminants influencent indépendamment ou concomitamment ou cumulativement l'évaluation du concept de soi de l'élève. Ils permettent de mieux connaitre l'élève grâce à l'identification des facteurs qui agissent 
prioritairement sur son sentiment de soi. Il serait alors possible d'agir sur ces facteurs pour amener l'élève à avoir sur lui-même, un jugement de valeur positif notamment l'amélioration des conditions de vie familiale (habitat, fourniture en électricité et eau, repas journaliers, etc.,) et des conditions de fréquentation (cantine, distance Ecole-famille). Il serait surtout possible pour le maître d'adapter son intervention aux caractéristiques particulières de l'élève grâce à une approche individualisée, ou à tout le moins, par focus group, dans le cadre d’une pédagogie différenciée.

\section{References:}

1. Bandura, A. (1977). Social learning theory, englewood, diff, NJ Prentice Hall.

2. Bandura, A. (1981). Self-referent thought: a developpement analysis of self efficaty, In $\mathrm{JH}$.

3. Bandura, A. (1986). Social foundation of thought and action: A social cognitive theory.Englewood Cliffs, NJ: Prenice Hall.

4. Boersma \& Chapman (1992). Persception of ability scale for students. Los Angeles, CA: western psychological service.

5. Bong, M. (1997). Generality of academic self-efficaty judgement: evidence of hierarchical relation. Journal of educational psychology, 89.

6. Bong, M. (1998). Test of the internal frames of reference model with subject academic journal of educational psychology.

7. Bressoux, P. (2003). Quand les enseignants jugent leurs élèves Paris, PUF.

8. Brière (1995). Développement et validation d'une mesure intrinsèque de l'échelle de motivation dans le sport. Journal of sport psychology.

9. Chanal, J. (2005). la formation du concept de soi en éducation physique et Sportive : les différents antécédents et rôle des visions de soi. Thèse de doctorat de l'université Jean Fourier, Grenoble Novembre 2005.

10. Chapman, D. (1991). Vision, instruction, and action. Cambridge, MA : MI Presse.

11. Colemen, S. (1966). Equal educational opportunity, Revue française de pédagogie $n^{\circ} 16,1971$ pp41-43.

12. Coopersmith, S. (1967). The antecedents of self-esteem. San Francisco, CA:W.H.freeman.

13. Dolata, S. (2008). Indice du statut socioéconomique du milieu familial des élèves du SACMEQ : construction avec le modèle de Rasch et analyses. Revue Mesure et Evaluation en Education vol. 31(1) pp121149. 
14. Dienrendonck, C. (2008). Validation psychométrique d'un questionnaire francophone de description de soi adapté aux préadolescents, Revue Mesure et Evaluation en,Education, Vol.31(1) pp51-91.

15. Dikes, P., Tournois, J., Flieller, A., \& Kop (1994). La psychométrie, Paris, PUF.

16. Gauthier, J., Samson, P., \& Turbide, D. (1981). Adaptation française du "social self- esteem inventory". Revue canadienne des sciences du comportement, 13, 218-225.

17. Hater, S. (1982). The perceived competence scale for children. Child development 53, 87, 97.

18. Hater, S. (1985). Competence as a dimension of self-evaluation towards a comprehensive model of self-worth. Orlando academic Press.

19. Heider, F. (1958). The psychology of interpersonnel relation, Oxfod wiley.

20. Harter, S. (1985). Manual for self-perception profile for children. Denver, CO : University of Denver.

21. Harter, S. (1993). Causes and consequences of low self-esteem in children and adolescents. IN R.F. Baumeister (éd.), self-esteem: the puzzle of low self-regard (pp.87-116). New York: Plenum Press.

22. Heyneman, S.P. \& Loxley, W.A. (1983). The effects of primary school quality on academic achievement across twenty-nine high-and-lowincome countries. American journal of sociology, 88(6) 1162-1194.

23. Kling, K.C., Hyde, J.S., Showers, C.J., \& Buuswell, B.N (1999). Gender differences in self-esteem; A meta-analysis. Psychological Bulletin, 125,470-500.

24. L'Ecuyer, R. (1981). The development of the self-concept through life.

25. Marsh, H. (1986). Multidimensional self-concept, Sex Roles.

26. Marsh, H. (1984). Determinant of students self-concept, journal of personality and social psychology 47, 213-231. Description de soi adapté aux préadolescents, Revue Mesure et Evaluation en Education, Vol.31(1) pp51-91. Span Paris, Edition du Seuil.

27. Marsh, H.W. (1988). Self-Description Questionnary: A theorical and empirical basis for the measurement of multiple dimensions of preadolescent self-concept. A test manual and a research monograph. San Antonio,TX: Psychological corporation.

28. Marsh, H.W. (1990). A multidimensionnal, hierarchical selfconcept:Theoritical and empirical justification. Educationnal Psychology review, 277-172.

29. Marsh, H.W. (1988). Self-Description questionnaire: A theoretical and empirical basis for measurement of multiple dimensions of 
preadolescent self-concept. A manual and a research monograph. San Antonio, TX: Psychological Corporation.

30. Marsh, H.W. (1993). Academic self-concept: Theory measurement and research. In J.Suls (éd.), Psychological perspectitive on the selfconcept (Vol.4pp.59-98) Hillsdale, NJ; Erlbaum.

31. Marsh, H.W. \& Ayotte, V. (2000). Developpment of an increasingly multidimensional self- concept for young children: French version of the self-description Questionnaire. Sydney: SELF Research Centre, University of Western Sydney.

32. Marsh, H.W. \& Ayotte, V. (2003). Do multiple dimensions of selfconcept become more differentiated with age? The differential distinctiveness hypothesis. Journal of Educational Psychology, 95, 687-706.

33. Marsh, H.W. \& Craven, R. (1997). Academic self-concept: beyond the dustbowl. In G. Phye (éd.) Handbook of classroom assessment: learning, achievement, and adjustment (pp.131-198). Orlando, FL: Academic Press.

34. Nino, G. \& Delignières, D. (2000). l'évaluation de l'estime de soi dans le domaine corporel. Revue S.T.A.P.S., 53,35-48.

35. Rosemberg, M. (1965). Society and the adolescent self-image. Princeton, NJ: Princeton University Press.

36. Shavelson, R.J., Hubner, J.J., \& Stanton, G.C. (1976). validation and construct interprétation. Review of Education Research. 46, 407-441.

37. Staats, A.W. (1975). Social Behaviorism. Homewood, IL: Dorsey Press.

38. Wylie, R.C. (1979). The self-concept Vol2 Lincoln, NE: University of Nebraska Press. 